ARTIGO ORIGINAL

\title{
Caracterização das propriedades mecânicas da madeira de clones de Hevea brasiliensis (Willd. Ex Adr.)
}

\author{
Characterization of mechanical properties of wood of clones of Hevea brasiliensis \\ (Willd. Ex Adr.)
}

Israel Luiz de Lima (D), Rafaela Bergamo², Karen Rizzato Bermudez², Mario Luiz Teixeira de Moraes ${ }^{3}$ (D) José Nivaldo Garcia² (1)

${ }^{1}$ Estação Experimental de Tupi, Instituto Florestal do Estado de São Paulo, Piracicaba, SP, Brasil

2Escola Superior de Agricultura Luiz de Queiroz, Universidade de São Paulo - USP, Piracicaba, SP, Brasil

${ }^{3}$ Faculdade de Engenharia do Campus de Ilha de Solteira - FEIS, Universidade Estadual Paulista "Júlio de Mesquita Filho" UNESP, Ilha Solteira, SP, Brasil

Como citar: Lima, I. L., Bergamo, R., Bermudez, K. R., Moraes, M. L. T., \& Garcia, J. N. (2020). Caracterização das propriedades mecânicas da madeira de clones de Hevea brasiliensis (Willd. ex Adr.). Scientia Forestalis, 48(125), e2877. https://doi.org/10.18671/scifor.v48n125.04

\section{Resumo}

A seringueira é uma espécie nativa do Brasil, cuja ocorrência é característica da floresta Amazônica, mas facilmente adaptável na maioria do território nacional. O principal uso da seringueira é a extração do látex, cujo ciclo é viável economicamente por 25 anos, e a madeira, após corte, é destinada geralmente para uso energético. Com o intuito de aproveitar a madeira industrialmente, este estudo visa selecionar clones de $\mathrm{H}$. brasiliensis, caracterizando as suas propriedades mecânicas. O experimento foi instalado em 1982 na Fazenda de Ensino, Pesquisa e Extensão da Faculdade de Engenharia de Ilha Solteira/UNESP, localizada em Selvíria, MS. O delineamento experimental utilizado foi o de blocos casualizados, compostos por cinco tratamentos (clones), com 5 repetições e parcelas lineares de 10 plantas, no espaçamento de $8 \mathrm{~m} \times 2,5 \mathrm{~m}$. Foram amostradas 25 árvores, cinco de cada clone, escolhidas dentro de cada bloco. Ao analisar as propriedades mecânicas nota-se variação entre os clones, onde se destaca o clone RRIM 600 pelos maiores valores, e os clones IAN 717 e o GT1 com os menores valores. Os resultados verificados podem subsidiar as informações para efeito comparativo com algumas espécies utilizadas comercialmente para fins estruturais.

Palavras-chave: Seringueira; Qualidade da madeira; Resistência mecânica.

\begin{abstract}
The rubber tree is a native species of Brazil, whose occurrence is characteristic of the Amazonian forest, but easily adaptable in most of the national territory. The main use of the rubber tree is the extraction of latex, whose cycle is economically viable for 25 years, and the wood, after cutting, is usually destined for energy use. In order to use it industrially, this study aims to select clones of $H$. brasiliensis, characterizing their mechanical properties of wood. The experiment was installed in 1982 at the Faculty of Engineering of Ilha Solteira / UNESP, located in Selvíria, MS. The experimental design was a randomized block, composed of five treatments (clones), with 5 replicates and linear plots of 10 plants, at a spacing of $8 \mathrm{~m} \times 2.5 \mathrm{~m}$. We sampled 25 trees, five of each clone, chosen within each block. When analyzing the mechanical properties, it is possible to observe a variation between the clones where the clone RRIM 600 is highlighted by the highest values, and the clones IAN 717 and GT1 with the lowest values. The results verified can subsidize the information for comparative effect with some species used commercially for structural purposes.
\end{abstract}

Keywords: Rubber; Quality of wood; Mechanical strength.

Fonte de financiamento: Conselho Nacional de Desenvolvimento Científico e Tecnológico - CNPq: Processo: 306539/2015-2.

Conflito de interesse: Nada a declarar.

Autor correspondente: limailde@gmail.com

Recebido: 3 maio 2018

Aceito: 6 maio 2019.

Editor: Francides Gomes da Silva Júnior.

(c) (i) Este é um artigo publicado em acesso aberto (Open Access) sob a licença Creative Commons Attribution, que permite uso, distribuição e reprodução c) em qualquer meio, sem restrições desde que o trabalho original seja corretamente citado. 


\section{INTRODUÇÃO}

No Brasil, espécies nativas são pouco cultivadas em plantios comerciais como é feito para as espécies do gênero Pinus e Eucalyptus. No entanto, o aumento no mundo da demanda por madeira tem levado a uma busca por novas espécies com potencial silvicultural e, neste cenário, espécies nativas podem ser vantajosas em relação sua adaptabilidade (Vidaurre et al., 2018).

Uma das espécies nativas do Brasil, que tem potencial para uso em reflorestamento, é a seringueira, adaptável a grande parte do território nacional, sendo considerada uma espécie arbórea de rápido crescimento (Pavezi \& Frazão, 1983). Hevea brasiliensis (seringueira) pertence à família Euphorbiaceae, as árvores dessa espécie podem atingir normalmente $20 \mathrm{a}$ $30 \mathrm{~m}$ de altura, com tronco desenvolvido, de $30 \mathrm{a} 60 \mathrm{~cm}$ de diâmetro, ereto e sem ramificações (Lorenzi, 2002).

Atualmente, a seringueira tem como principais locais de cultivo no Brasil, as denominadas áreas de escape do mal-das-folhas (doença causada pelo fungo Microcyclus ulei), e localizam-se nos estados de São Paulo, Mato Grosso, Mato Grosso do Sul, Bahia e no noroeste do Paraná (Pereira, 1992). As condições climáticas adequadas, que minimizam os riscos de insucesso, e também os altos rendimentos proporcionados pela cultura nos últimos anos, são os principais fatores que elevaram São Paulo à condição de maior produtor de borracha natural desde 1995, respondendo por aproximadamente metade da produção total no país (Gonçalves et al., 2001). A área plantada com seringueira, no Brasil, vem aumentando significativamente, de 159.000 ha em 2010, para 229.964 ha em 2016 (Indústria Brasileira de Árvores, 2017).

As árvores de seringueira no final da sua vida produtiva apresentam um perímetro médio do caule (PMC) em torno de 110-100 cm (125 cm acima do solo), sendo aptas para corte aproximadamente 184 árvores/hectare. De uma árvore obtém-se $0,62 \mathrm{~m}^{3}$ provenientes do tronco e cerca de $0,39 \mathrm{~m}^{3}$ provenientes dos ramos laterais, totalizando $1,10 \mathrm{~m}^{3} / a ́ r v o r e$ (Pushpadas et al., 1980). Para Peries (1990), há possibilidade de extração de $210-260 \mathrm{~m}^{3}$ de madeira/ha em um seringal, no final do seu ciclo produtivo.

Jacovine et al. (2006) argumentam que a $H$. brasiliensis sendo uma espécie arbórea de crescimento rápido, tem capacidade de fixação de carbono, transformando-o em látex, madeira, etc., o que contribui para a minimização dos problemas ambientais. Após a implantação, de acordo com esses autores, o plantio de seringueira constitui em um sistema estável com características de uma floresta tropical, que conserva o solo, melhora o meio ambiente e ainda pode gerar empregos e riquezas.

Quando as árvores de seringueira não atingem mais níveis aceitáveis de produção de látex, que varia entre 25 a 30 anos de idade, se torna economicamente inviável sua exploração; nesse momento essas árvores são abatidas para a reformulação do plantio, sendo a madeira utilizada normalmente como combustível (Kamala \& Rao, 1989).

Para Ramos et al. (2016), o Brasil não tem tradição no uso industrial da madeira de seringueira, a não ser para produção de biomassa. Entretanto, a mesma poderia ser utilizada na fabricação de portas, janelas, formas para concreto armado, vigas, colunas, painéis e artigos domésticos na forma de madeira compensada (Haridasan, 1989). Segundo Okino et al (2004), a madeira de seringueira in natura se mostrou tecnicamente viável à produção de chapas de madeira para uso com cimento, independentemente do clone cultivado.

A madeira de $H$. brasiliensis é considerada leve, mole, de baixa durabilidade natural e de alburno indistinto, apresenta densidade em torno de 560 a $650 \mathrm{~kg} \cdot \mathrm{m}^{-3}$, e a umidade da madeira recém-cortada é de aproximadamente $60 \%$, podendo ser reduzida para $15 \%$ quando seca ao ar, exigindo pelo menos 10 dias de exposição nessas condições (Lorenzi, 2002).

O grande problema da utilização dos produtos provenientes da madeira de seringueira é a alta susceptibilidade ao ataque de fungos e insetos (besouros e cupins), devido à ausência de cerne na madeira e a um alto teor de amido e açúcares necessitando, portanto, de um tratamento profilático logo após o corte, em um período menor que 24 horas (Peries, 1990). A recomendação de tratamento químico é a mesma para outras madeiras em geral, e a escolha do tratamento mais adequado depende da finalidade a que se destina o material (May \& Gonçalves, 2014). 
Segundo Ramos et al. (2016), a presença de tensão na madeira de seringueira já é bem conhecida, e vários estudos já foram desenvolvidos para tentar amenizar os problemas que relaciona este fato com a baixa durabilidade e a instabilidade dimensional.

Em relação à variação radial das propriedades da madeira $H$. brasiliensis, não foram encontrados na literatura muitos artigos que permitam fazer avaliações específicas para esta espécie. Porém, de maneira geral, é esperado que ocorra um aumento dos valores da resistência mecânica, no sentido medula-casca (Leonello et al., 2012).

De modo geral verificamos que estudos sobre qualidade de madeira de $H$. brasiliensis são ainda escassos, principalmente no que se refere a sua utilização para a produção de madeira serrada e que visem também maximizar e elevar a renda dos produtores que a cultivam.

Os clones RRIM 600 e GT 1 são os principais comercialmente utilizados atualmente no Brasil, os mesmos podem ter aplicações em pequenas estruturas, construção leve, e indústria de móveis, podendo ser uma alternativa a ser utilizada para o mercado madeireiro (Viégas et al., 2015).

Eufrade Junior et. ali. (2015) afirmam que para ser ter um melhor uso da madeira de seringueira e obter melhor vantagem e de forma mais eficaz em aplicações de engenharia, as características específicas e as propriedades físicas e mecânicas devem ser consideradas.

Sendo assim, o objetivo desse trabalho foi caracterizar as propriedades mecânicas da madeira de genótipos (clones) de $\mathrm{H}$. brasiliensis e ainda fornecer subsídios básicos para o melhor aproveitamento industrial desta madeira.

\section{MATERIAL E MÉTODOS}

O material de estudo teve como base uma população de Hevea brasiliensis, composta de clones selecionados procedentes do Polo Regional de Votuporanga, SP. Esta população encontra-se instalada em Selvíria, MS. O relevo é caracterizado por ser moderadamente plano e ondulado. O clima do local é do tipo Aw pela classificação de Köppen, com temperatura média anual de $23^{\circ} \mathrm{C}$, precipitação média anual de $1440 \mathrm{~mm}$ (Instituto de Pesquisas e Estudos Florestais, 2018). O solo local é classificado como Latossolo Vermelho distrófico (LVd) típico argiloso, A moderado, hipidistrófico, álico, caulinítico, férrico, compactado, muito profundo, moderadamente ácido (Santos et al., 2006).

$O$ teste de clone de seringueira foi instalado em 02/03/1982. Os clones utilizados foram: LCB 510, RRIM 600, IAM 717, IAN 873 e GT 1 (testemunha). O delineamento experimental utilizado foi o de blocos casualizados, compostos por 5 tratamentos (clones), 5 repetições e parcelas lineares de 10 plantas, no espaçamento de 8,0 × 2,5 metros.

Porém, para o estudo das propriedades da madeira foram amostradas 25 árvores, sendo uma de cada clone, escolhida dentro de cada bloco. As árvores foram selecionadas na classe de DAP média, em cada parcela (Tabela 1).

Tabela 1. Idade, diâmetro a altura do peito (DAP) e altura total (HT) dos clones.

\begin{tabular}{ccccc}
\hline Tratamentos & Clone & $\begin{array}{c}\text { Idade } \\
\text { (anos) }\end{array}$ & $\begin{array}{c}\text { DAP } \\
(\mathbf{c m})\end{array}$ & $\begin{array}{c}\text { HT } \\
(\mathbf{m})\end{array}$ \\
\hline 1 & LCB 510 & 33 & 25,20 & 16,94 \\
2 & RRIM 600 & 33 & 24,10 & 18,76 \\
3 & IAN 873 & 33 & 23,30 & 17,52 \\
4 & IAN 717 & 33 & 27,00 & 20,94 \\
5 & GT 1 & 33 & 25,00 & 18,76 \\
\hline
\end{tabular}

Antes da derrubada das árvores foi marcada a direção norte em cada uma delas. Das árvores escolhidas foi tomada a primeira tora de $1 \mathrm{~m}$ de comprimento, adequadamente identificadas e marcadas. Posteriormente, essas toras foram transportadas para o Laboratório de Engenharia da Madeira da ESALQ/USP, Piracicaba, SP, onde as mesmas foram desdobradas. 
Em cada uma das toras desdobradas foram tiradas uma prancha central $7 \mathrm{~cm}$ de espessura. Das pranchas, foram retirados três sarrafos de $4 \mathrm{~cm} \times 4 \mathrm{~cm} \times 1 \mathrm{~m}$, sendo os mesmos representativos das posições $(0,50$ e 100\%) do raio da tora, dos quais foram retirados os corpos de prova específicos para cada ensaio físico-mecânico, realizados no Laboratório de Engenharia da Madeira da ESALQ/USP.

\section{Propriedades Mecânicas da madeira}

Todos os ensaios mecânicos foram feitos com amostras secas, em ambiente com temperatura normalizada, até atingirem, aproximadamente, 12\% de umidade, condição de referência padrão, de acordo com a recomendação da NBR7190 (Associação Brasileira de Normas Técnicas, 1997).

a) Resistência à compressão paralela às fibras $\left(\mathrm{f}_{\mathrm{co}}\right)$

Para se obter a resistência à compressão paralela às fibras $\left(\mathrm{f}_{\mathrm{c} 0}\right)$, foram utilizados corpos de prova de $2 \times 2 \times 3 \mathrm{~cm}$, obtidos de cada sarrafo, num total de 75 unidades. Ensaios de compressão foram realizados em uma máquina universal de ensaio MB26 (Abnt, 1940), onde foi utilizada a mesma taxa de aplicação de tensão (taxa de carregamento da norma NBR 7190) que é de $10 \mathrm{MPa} / \mathrm{min}$ e, portanto, já considerada a área de carregamento (Associação Brasileira de Normas Técnicas, 1997).

Para a obtenção dos valores dessa variável foi utilizada a Equação 1.

$$
f_{c 0}=\frac{P_{r}}{A}
$$

Em que:

$\left(\mathrm{f}_{\mathrm{co}}\right)$ : resistência à compressão paralela às fibras, $\mathrm{MPa}$;

Pr: carga de ruptura, N, e

A: área da seção transversal, $\mathrm{mm}^{2}$.

b) Resistência ao cisalhamento tangencial aos anéis de crescimento $\left(f_{v}\right)$

$\mathrm{Na}$ obtenção da resistência ao cisalhamento tangencial $\left(f_{\mathrm{v} 0}\right)$ aos anéis de crescimento foi utilizado corpos de prova com dimensões nominais de $2 \times 2 \times 3 \mathrm{~cm}$, com $4 \mathrm{~cm}^{2}$ na área de cisalhamento. A velocidade de aplicação de carga foi de 2,5 $\mathrm{MPa}$ (N/mm²/min), a partir disso, a resistência ao cisalhamento foi definida pela máxima tensão de cisalhamento atuante no corpo de prova, como descrito na norma NBR 7190 (Associação Brasileira de Normas Técnicas, 1997) sendo determinada pela equação 2:

$$
f_{v o}=\frac{F_{v o 1 \max }}{A_{v}}
$$

Em que:

$\mathrm{f}_{\text {vo max: }}$ máxima força cisalhante aplicada ao corpo de prova $(\mathrm{N})$, e

$A_{v:}$ área inicial da seção crítica do corpo de prova, num plano paralelo as fibras ( $\left.\mathrm{m}^{2}\right)$.

c) Resistência a ruptura na flexão estática

Para resistência da madeira à flexão estática $\left(f_{M}\right)$ foram utilizados corpos de prova de $2 \times 2 \times 35 \mathrm{~cm}$, obtidos de cada sarrafo. Os ensaios foram conduzidos em uma máquina universal de ensaio com velocidade de aplicação de carga de $10 \mathrm{MPa}$. Foi utilizada uma combinação da norma MB 26 e a ASTM D143-94 (American Society of Testing and Materials, 1994) com dimensões ( $b \times h)$ de $2 \times 2 \mathrm{~cm}$ para seção transversal e $30 \mathrm{~cm}$ de vão livre $(\mathrm{L})$ redundando na relação $\mathrm{L} / \mathrm{h}$ igual a 15. Essa relação está acima da atualmente recomendada pela ASTM D143-09 (American Society of Testing and Materials, 2009) para a determinação do módulo de elasticidade na flexão estática que é de 14. Para esta variável foi utilizada a Equação 3. 


$$
f_{M}=\frac{3 P_{r} L}{2 b h^{2}}
$$

Em que:

$\mathrm{f}_{\mathrm{M}}$ : resistência à flexão estática, $\mathrm{MPa}$;

$\mathrm{P}_{\mathrm{r}}$ : carga de ruptura, $\mathrm{N}$;

L: vão livre (fixado em $30 \mathrm{~mm}$ );

b: lado da seção transversal do corpo de prova, perpendicular à força aplicada, mm, e

h: lado da seção transversal do corpo de prova paralelo à força aplicada, mm.

d) Modulo de elasticidade na flexão estática

O modulo de ruptura e elasticidade na flexão estática foram testados simultaneamente com ensaios em corpo de prova de $(2 \times 2 \times 35) \mathrm{cm}$, retirados de cada sarrafo. O ensaio foi conduzido na mesma máquina universal. O módulo de elasticidade na flexão estática (Ef) foi obtido pela Equação 4:

$$
E_{f}=\frac{B L_{3}}{4 b h_{2}}
$$

Em que:

Ef: módulo de elasticidade na flexão estática $\mathrm{m}^{2}$;

B: coeficiente angular da reta a ser encontrado através da regressão linear da equação: $\mathrm{P}=\mathrm{A}+\mathrm{Bf} \mathrm{N} / \mathrm{m}^{2}$;

$\mathrm{L}$ : vão livre do corpo de prova, dimensão nominal de 0,30m;

b: base da seção transversal, dimensão nominal de 0,02m, e

h: altura da seção transversal, dimensão nominal de 0,02m.

\section{Propriedade Física da Madeira}

a) Densidade aparente $(\rho \mathrm{ap})$

Amostras de $(2 \times 2 \times 3) \mathrm{cm}$ foram obtidas dos sarrafos representativos das diferentes posições do tronco da arvores e secas até atingirem 12\% de umidade, para a obtenção da densidade aparente. As dimensões das amostras foram aferidas com paquímetro digital com sensibilidade de $0,01 \mathrm{~cm}$, e a massa do corpo de prova foi obtida em balança semianalítica, com sensibilidade de 0,01 g MB26 (Associação Brasileira de Normas Técnicas, 1940). Para a obtenção do valor da massa específica foi utilizada a Equação 5.

$$
\rho_{a p}=\frac{P_{u}}{V_{u}}
$$

Em que:

$\rho_{\mathrm{ap}}$ : densidade aparente, $\mathrm{g} \cdot \mathrm{cm}^{-3}$;

$\mathrm{P}_{\mathrm{u}}$ : massa do corpo de prova a $12 \%$ de umidade, $\mathrm{g}$, e

$\mathrm{V}_{\mathrm{u}}$ : volume do corpo de prova a $12 \%$ de umidade, $\mathrm{cm}^{3}$.

\section{Análise estatística}

A avaliação do experimento foi efetuada utilizando-se modelos matemáticos, que foram ajustados para considerar a variação completa de todos os tratamentos. Inicialmente foi efetuado o teste de homogeneidade de variância e, para isso, utilizou-se o teste de Hartley. Posteriormente, foi feito $\mathrm{o}$ teste $\mathrm{F}$ de análise de variância segundo o delineamento experimental em blocos casualizados, adotando-se o esquema fatorial $5 \times 3$ (clone x posição radial). Foi aplicado o teste de Tukey, sempre que foi observado diferença significativa, ao nível 
de $5 \%$ de probabilidade, de algum tratamento no teste F. Os dados obtidos para cada variável foram analisados estatisticamente com o auxílio do procedimento estatístico PROC GLM do SAS (SAS Institute Inc., 1999).

\section{RESULTADOS E DISCUSSÃO}

Os resumos das análises de variância para as variáveis resistência à compressão paralela às fibras $\left(f_{c 0}\right)$, resistência à flexão estática $\left(f_{M}\right)$, módulo de elasticidade na flexão estática $\left(E_{M 0}\right)$, resistência ao cisalhamento paralela às fibras $\left(f_{\text {vo }}\right)$ e densidade aparente $\left(\rho_{a p}\right)$ estão apresentados na Tabela 2. De acordo com os resultados verifica-se que ocorreram diferenças significativas entre os clones. Porém, para a posição radial somente ocorreu variações significativas para o módulo de elasticidade na flexão estática $\left(\mathrm{E}_{\mathrm{MO}}\right)$. Não ocorreu interação significativa entre clones e posição radial, o que demostra que esses fatores não tem dependência entre si (Tabela 2).

Tabela 2. Resumo da análise de variância efetuada para resistência à compressão paralela às fibras $\left(f_{\mathrm{co}}\right)$, resistência à flexão estática $\left(\mathrm{f}_{\mathrm{M}}\right)$, módulo de elasticidade na flexão estática $\left(\mathrm{E}_{\mathrm{MO}}\right)$, resistência ao cisalhamento paralela às fibras $\left(f_{\mathrm{vo}}\right)$ e densidade aparente $\left(\rho_{\mathrm{ap}}\right)$ de Hevea brasiliensis de 33 anos.

\begin{tabular}{|c|c|c|c|c|c|c|}
\hline \multirow[b]{2}{*}{ Causa de Variação } & \multirow[b]{2}{*}{ GL } & \multicolumn{5}{|c|}{ Quadrado Médio } \\
\hline & & $\begin{array}{c}\rho_{a p} \\
\left(\mathrm{~g} \mathrm{~cm}^{-3}\right)\end{array}$ & $\begin{array}{c}\mathbf{f}_{\mathrm{c0}} \\
(\mathrm{MPa})\end{array}$ & $\begin{array}{c}\mathbf{f}_{\mathrm{v} 0} \\
(\mathrm{MPa})\end{array}$ & $\begin{array}{c}\mathbf{f}_{\mathbf{M}} \\
(\mathbf{M P a})\end{array}$ & $\begin{array}{c}\mathrm{E}_{\mathrm{MO}} \\
(\mathrm{MPa})\end{array}$ \\
\hline Clone (C) & 4 & $0,0245 * *$ & $270,49 * *$ & 11,59 * & $1933 * *$ & 35908037 ** \\
\hline Posição radial (P) & 2 & $0,0073^{\text {n.s. }}$ & 26,81 n.s. & 2,99 n.s. & $1147^{\text {n.s. }}$ & 41716363 * \\
\hline$C \times P$ & 8 & $0,0017^{\text {n.s. }}$ & $10,75^{\text {n.s. }}$ & $2,09^{\text {n.s. }}$ & $320^{\text {n.s. }}$ & $4354635^{\text {n.s. }}$ \\
\hline Resíduo & 60 & 0,0012 & 37,74 & 4,32 & 405 & 8388043 \\
\hline Média & & 0,61 & 43,23 & 12,51 & 75,76 & 10043 \\
\hline $\mathrm{CV}_{\mathrm{e}}$ & & 5,68 & 14,21 & 16,63 & 26,59 & 28,84 \\
\hline
\end{tabular}

Onde: ** significativo ao nível de $1 \%$ de significância; * significativo ao nível de $5 \%$ de significância e n.s. = não significativo; $\mathrm{CV}_{\mathrm{e}}=$ coeficiente de variação experimental.

No ensaio de densidade aparente, o clone RRIM 600 diferiu significativamente dos demais, apresentando o maior valor, enquanto que o IAN 717 apresentou o menor valor (Figura 1). O valor médio da densidade da madeira de seringueira verificado foi $0,61 \mathrm{~g} . \mathrm{cm}^{-3}$ (Tabela 1). Este valor é superior aos encontrados por Riyaphan et al. (2015) para o RRIM 600, com 13 anos de idade. Naji et al. (2012) verificaram valores de 0,57 e 0,61 g. $\mathrm{cm}^{-3}$ para o RRIM 2020 e RRIM 2025, ambos com 9 anos de idades. Santana et al. (2001) constataram valor médio de 0,63 g. $\mathrm{cm}^{-3}$ para quatro clones de seringueira, porém com idade de 38 anos. As densidades constatadas para todos os clones podem ser classificadas como sendo de madeira moderadamente pesada, de classe de resistência 20 (C20) NBR 7190 (Associação Brasileira de Normas Técnicas, 1997). 


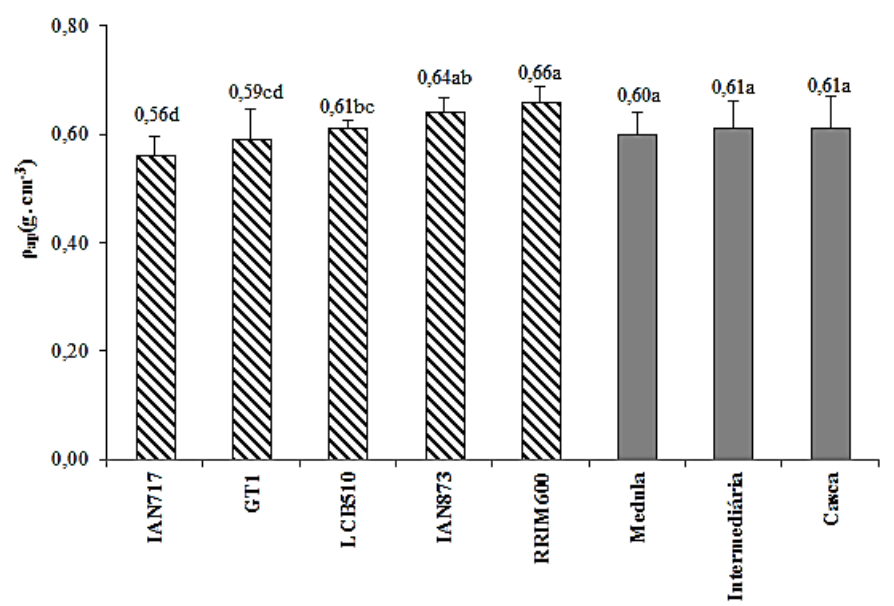

Figura 1. Densidade aparente ( $\left.\rho_{a p}\right)$ em função do clone e da posição radial de Hevea brasiliensis de 33 anos. Obs: Médias seguidas da mesma letra não diferem entre si pelo teste de Tukey a $5 \%$ de probabilidade.

Segundo as análises estatísticas efetuadas, tem-se que o clone RRIM 600 diferiu significativamente dos clones IAN 717 e do GT1 para a compressão paralela às fibras (Figura 2a). O valor médio apresentado foi de 43,23 MPa (Tabela 2). Este valor médio é semelhante aos encontrados por Santana et al. (2001) para clones de seringueira e por Eufrade Junior et al. (2015) para o clone RRIM 600 de 29 anos de idade. De acordo com os valores constatados para a resistência da madeira (Figura 2), com exceção do clone GT1, todos os outros clones podem ser classificados como sendo da classe de resistência 40 (C40) NBR 7190 (Associação Brasileira de Normas Técnicas, 1997). Esses valores obtidos podem ser comparados com os da espécie Erisma uncinatum (cedrinho) que é muito utilizada na construção civil em estrutura leve e outros usos tais como: móveis, laminados, compensados, embalagens e caixas (Zenid, 2009). 
a)
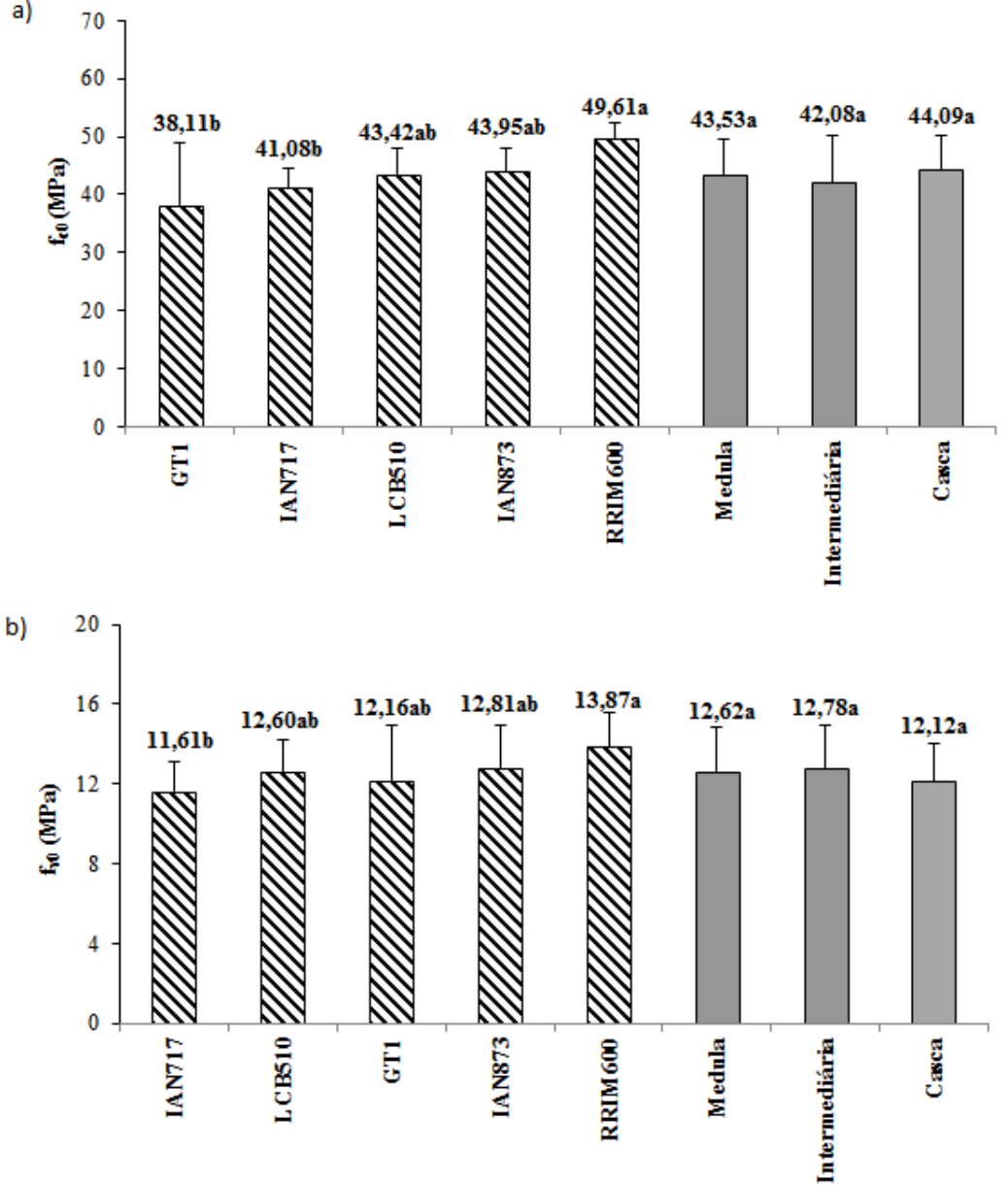

Figura 2. Compressão paralela às fibras $\left(f_{c 0}\right)$ e resistência ao cisalhamento paralela às fibras $\left(f_{v 0}\right)$ em função do clone e da posição radial de Hevea brasiliensis de 33 anos.

Para a resistência ao cisalhamento paralela às fibras, o RRIM 600 diferiu do IAN 717 (Figura 2b). Pode-se destacar o clone RRIM 600 com os maiores valores, e o clone IAN $717 \mathrm{com}$ os menores. Estes valores obtidos são similares aos que foram verificados por Santana et al. (2001) para clones de seringueira, porém são maiores do que verificados por Eufrade Junior et al. (2015) para o RRIM 600 e GT1.

Os testes estatísticos indicaram diferença significativa no desempenho do clone RRIM 600 em relação aos clones IAN 717 e GT1, para o ensaio de resistência à flexão estática (Figura 3a). Para o módulo de elasticidade na flexão estática, o clone RRIM 600 também se diferenciou significativamente dos clones GT1 e IAN 717 (Figura 3b) 
a)
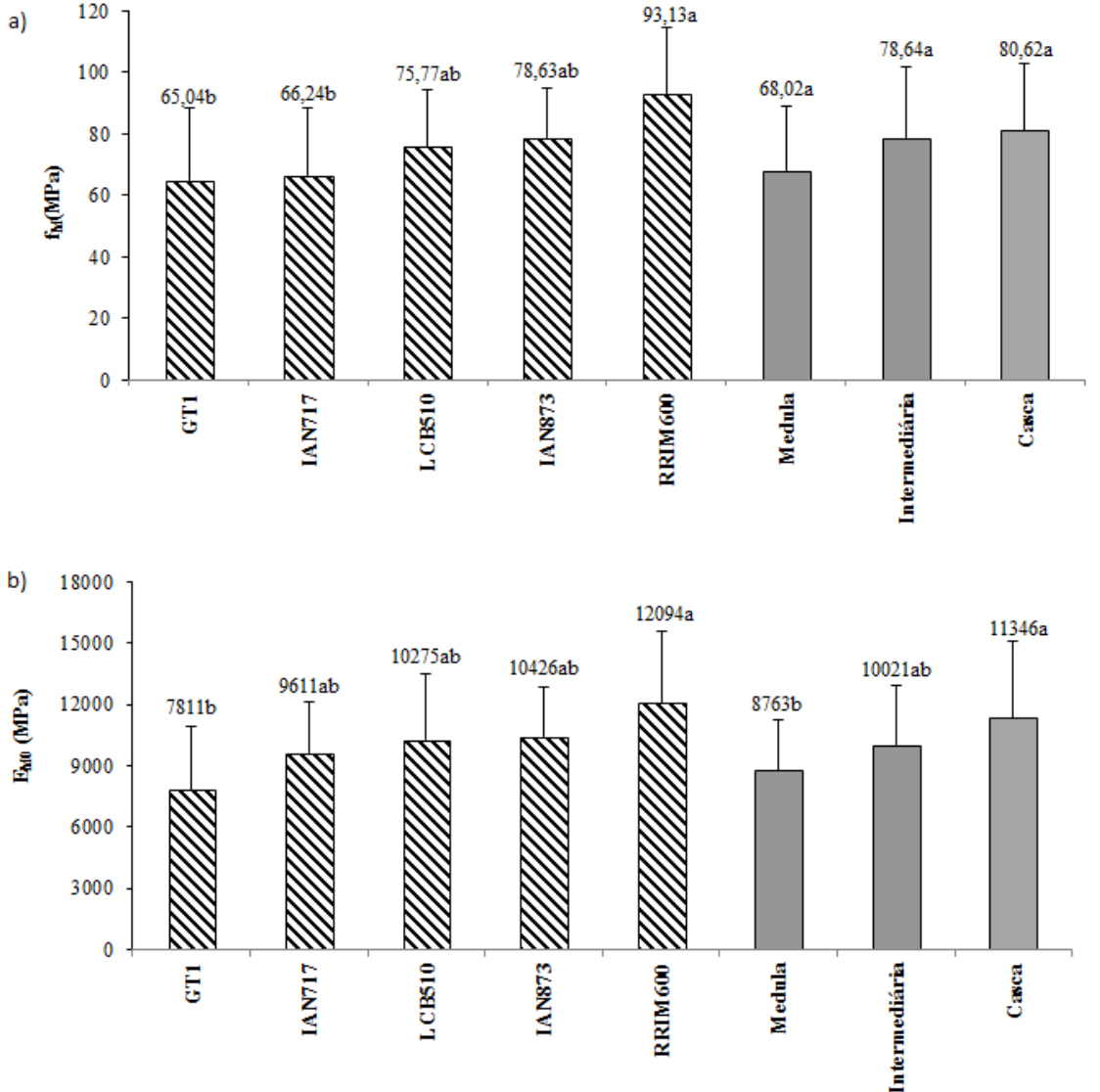

Figura 3. Resistência à flexão estática $\left(f_{M}\right)$ [a] e módulo de elasticidade na flexão estática $\left(\mathrm{E}_{\mathrm{MO}}\right)[\mathrm{b}]$ em função do clone e da posição radial de Hevea brasiliensis de 33 anos.

O clone RRIM 600 também se destacou para a resistência à flexão estática $\left(f_{M}\right)$ e módulo de elasticidade na flexão estática $\left(E_{M_{0}}\right)$, apresentando uma resistência significativamente maior do que os outros clones de $\mathrm{H}$. brasiliensis de 33 anos (Figura $3 a$ e 3b). Destaca-se que os clones IAN 717 e do GT1 apresentaram menor resistência, para ambas as propriedades (Figuras 3a e 3b). Riyaphan et al. (2015) obtiveram valores de 108 e $10.400 \mathrm{MPa}$ para $\left(f_{\mathrm{M}}\right)$ e $\left(E_{M 0}\right)$, respectivamente, para o clone de RRIM 600 , sendo que o $\left(f_{M}\right)$ foi maior e o $\left(E_{M 0}\right)$ foi menor, do que os obtidos neste trabalho.

De maneira geral, a densidade aparente, a resistência à compressão paralela às fibras, o módulo de elasticidade na flexão estática e a resistência ao cisalhamento paralela às fibras foram influenciados significativamente pelos clones, onde se pode destacar, o clone RRIM 600 que apresentou os valores mais altos para todas as propriedades em estudo, enquanto que os clones IAN 717 e o GT 1 apresentaram os menores valores em quase todas as propriedades (Figuras 1, 2 e 3). Esses valores de resistência mecânica dos clones da seringueira obtidos são maiores do que Oliveira et al. (2006) constataram para Pinus taeda de 20 anos, sendo, então, um indício de que a madeira da seringueira pode ter algumas utilidades que é dada à madeira de Pinus usada comercialmente no Brasil.

Para Gonçalves et al. (1991), no estado de São Paulo os clones RRIM 600, GT 1 e IAN 873 são uns dos mais utilizados em plantios comerciais e, ainda, destacam que o clone RRIM 600 normalmente apresenta os maiores desenvolvimento silvicultural e maior disponibilidade no mercado. De acordo com os resultados das propriedades verificados, podemos então, indicar o uso da madeira do RRIM 600 para pequenas estruturas. Gonçalves et al. (2001) estima, também, que o clone RRIM 600 seja o mais plantado, com cerca de $80 \%$ da área paulista utilizada para o cultivo de seringueira. Segundo Eufrade Junior et al. (2015) considerando o mercado brasileiro de madeiras nativas, os clones RRIM 600 e GT1 têm potencial para substituir algumas madeiras comerciais, especialmente Cedrela spp. (Cedro espanhol), Cedrelinga cateniforme (Tornillo), Vochysia spp. (Quaruba) e Erisma uncinatum (cedrinho), sendo 
que esta última espécie está entre as madeiras que são mais comercializadas e exploradas a partir da floresta Amazônica atualmente.

A densidade aparente não se diferenciou significativamente entre as posições radiais (Figura 1). A compressão e o cisalhamento não variaram significativamente entre as posições radiais (Figuras $2 \mathrm{a}$ e $2 \mathrm{~b}$ ). Também, a resistência à flexão estática não variou significativamente na posição radial (Figura 3a), enquanto que para o módulo de elasticidade na flexão estática houve um aumento no sentido da medula para casca (Figura 3b). Com relação a essa variação medula-casca constatada, as amostras não apresentaram um padrão típico de variação para a maioria das propriedades mecânicas, e também, para a densidade, a única exceção foi no ensaio de módulo de elasticidade na flexão estática $\left(\mathrm{E}_{\mathrm{MO}}\right)$, onde ocorreu um aumento significativo no sentido da medula para casca. Resultados similares foram obtidos por Naji et al. (2012), onde verificaram um aumento da densidade da madeira nos primeiros anéis próximo da medula e uma diminuição nas proximidades da casca. Umas das explicações, para esses clones não apresentarem um padrão típico de variação radial das propriedades em estudo seria o fato da seringueira ser propagada pelo método de enxertia, isso, pode ter alterado a fisiologia de crescimento da planta, causando um deslocamento da medula.

Severo et al. (2013) também verificaram em madeira de $H$. brasiliensis que não há diferença significativa na densidade, entre madeira juvenil e adulta, relatam que isto é explicado pelos altos níveis de conteúdo de extrativos observado na madeira juvenil. Leonello et al. (2012) verificaram para o clone GT1 que no geral não houve diferença estatisticamente significativa da variação de propriedades mecânicas analisadas na direção radial, porém houve tendência de aumento no valor dessas propriedades no sentido medulacasca, e ainda não houve grande influência da juvenilidade nas propriedades mecânicas, com exceção da dureza Janka, que apresentou como comportamento o aumento de seus valores no sentido medula-casca.

Constatou-se então, neste trabalho, que a madeira de seringueira pode ter uma melhor utilização industrial. Sugere em futuros de plantios de seringueira com finalidade de produção de madeira e látex, sejam selecionados clones que produzam mais látex, aliado com a quantidade e a qualidade da madeira a ser produzida. Dessa maneira, o reflorestamento utilizando seringueira pode ser mais uma alternativa de produção de madeira de forma consciente e sustentável.

\section{CONCLUSÃO}

Existe variabilidade significativa nas propriedades mecânicas e densidade aparente da madeira de diferentes clones de $H$. brasiliensis.

Não existem variações significativas, em função da posição radial, no tronco de H. brasiliensis. A única exceção foi para o módulo de elasticidade na flexão estática.

O material RRIM 600 apresenta maior resistência para todos os ensaios físicos-mecânicos, enquanto que os clones IAN 717 e o GT1 apresentam as menores resistência.

O clone RRIM 600 tem o mesmo potencial de utilização comercial que possui a madeira de Pinus taeda e Erisma uncinatum (cedrinho), desde que, passe por um tratamento preventivo contra os agentes xilófagos da madeira.

Considerando os valores das propriedades obtidas para os clones, esses podem ser utilizados para fins comparativos com as espécies utilizadas atualmente para fins estruturais no Brasil.

\section{AGRADECIMENTOS}

Os autores agradecem a José Cambuim (UNESP / Ilha Solteira) e Eduardo Luiz Longui (Instituto Florestal) pelo apoio na coleta de material de campo. Os autores Israel Luiz de Lima e Mario Luiz Teixeira de Moraes agradecem ao Conselho Nacional de Desenvolvimento Científico e Tecnológico - CNPq pela concessão da bolsa de Produtividade em Pesquisa e também pelas bolsas de iniciação cientifica concedidas às estagiárias Rafaela Bergamo e Karen Rizzato Bermudez. 


\section{REFERÊNCIAS BIBLIOGRÁFICAS}

American Society of Testing and Materials - ASTM. (1994). ASTM D 143-94: standard methods of testing small clear specimens timber. Philadelphia: ASTM.

American Society of Testing and Materials - ASTM. (2009). ASTM D 143-09: test methods for small clear specimens of timber. Philadelphia: ASTM.

Associação Brasileira de Normas Técnicas - ABNT. (1940). Ensaios físicos e mecânicos de madeira. MB 26/40. Rio de Janeiro: ABNT.

Associação Brasileira de Normas Técnicas - ABNT. (1997). Projeto de estruturas de madeira: projeto NBR 7190/1997. Rio de Janeiro: ABNT.

Eufrade Junior, H. J., Ohto, J. M., Silva, L. L., Lara Palma, H. A., \& Ballarin, A. W.. (2015). Potential of rubberwood (Hevea brasiliensis) for structural use after the period of latex extraction: a case study in Brazil. Journal of Wood Science, 61(4), 384-390. http://dx.doi.org/10.1007/s10086-015-1478-7.

Gonçalves, P. D. S., Bataglia, O. C., Ortolani, A. A., \& Fonseca, F. S. (2001). Manual de Heveicultura para o Estado de São Paulo (Série Tecnologia APTA, 189, 78 p.). Campinas: Instituto Agronômico.

Gonçalves, P. D. S., Cardoso, M., Boaventura, M. A. M., Colombo, C. A., \& Ortolani, A. A. (1991). Clones de Hevea: influência dos fatores ambientais na produção e recomendação para o plantio. (Boletim Técnico IAC, 138, 32 p.). Campinas: Instituto Agrônomico.

Haridasan, V. (1989). Rubber wood. promise of the future. Rubber Board Bulletin, 25, 7-8.

Indústria Brasileira de Árvores - IBÁ. (2017). Relatório Ibá 2017 (80p.). São Paulo: IBA.

Instituto de Pesquisas e Estudos Florestais - IPEF. (2018). Eucalyptus no Brasil - zoneamento climático e guia para identificação aptidão por município. Recuperado em 17 de abril de 2018, de http://www.ipef.br/publicacoes/guiaeucalyptus/aptidao.aspx

Jacovine, L. A. G., Nishi, M. H., Silva, M. L., Valverde, S. R., \& Alvarenga, A. P. (2006). Seringueira no contexto das negociações sobre mudanças climáticas globais. In A. P. Alvarenga, \& C. A. F. S. Carmo (Eds.), Sequestro de carbono: quantificação em seringais de cultivo e na vegetação natural (pp. 201 214). Viçosa: .

Kamala, B. S., \& Rao, P. V. K. (1989). Physical and mechanical properties of Hevea brasiliensis (rubber wood) - a review. Rubber Board Bulletin, 25, 27-28.

Leonello, E. C., Ballarin, A. W., Ohto, J. M., Palma, H., \& Escobar, J. F. (2012). Classificação estrutural e qualidade da madeira do clone GT 1 de Hevea brasiliensis Muell. Arg. Floresta e Ambiente, 19(2), 229235. http://dx.doi.org/10.4322/floram.2012.027.

Lorenzi, H. (2002). Árvores Brasileiras: manual de identificação e cultivo de plantas arbóreas nativas do Brasil (Vol. 1, 4. ed., 368 p.). Nova Odessa: Editora Plantarum.

May, A., \& Gonçalves, P. S. (2014). Produtos complementares na Exploração do Seringal - Matéria técnica. Borracha Atual, 17-21. Recuperado em 8 de março de 2014, de www.borrachaatual.com.br/materiatecnica/20/materia_tecnica_20a

Naji, H. R., Sahri, M. H., Nobuchi, T. Y., \& Bakar, E. S. (2012). Clonal and planting density effects on some properties of rubber wood (Hevea brasiliensis Müell. Arg.). BioResources, 7(1), 189-202.

Okino, E. Y. A., Souza, M. R., Santana, M. A. E., Sousa, M. E., \& Teixeira, D. E. (2004). Chapa Aglomerada de Cimento-madeira de Hevea brasiliensis Müll. Arg. Revista Árvore, 28(3), 451-457. http://dx.doi.org/10.1590/S0100-67622004000300016.

Oliveira, F. L., Lima, I. L., Garcia, J. N., \& Florsheim, S. M. B. (2006). Propriedades da madeira de Pinus taeda L. em função da idade e da posição radial na tora. Revista do Instituto Florestal, 18(2), 59-70.

Pavezi, R. T., \& Frazão, D. A. C. (1983). Características botânicas do gênero Hevea. Nutrição e adubação da seringueira no Brasil (116 p.). Campinas: Fundação Cargill.

Pereira, J. P. (1992). Seringueira: formação de mudas, manejo e perspectivas no noroeste do Paraná (Circular Técnica, 70, 60 p.). Londrina: IAPAR.

Peries, O. S. (1990). Rubber wood - a byproduct of the natural rubber industry. Rubber Research Institute of Sri Lanka Bulletin, 15, 1-5.

Pushpadas, M. V., Haridasan, V., \& Jayarathnam, K. (1980). By-products and ancillary in rubber plantations. In P. N. Radhakrishna Pillay (Ed.), Handbook of natural rubber production in India (pp. 505-514). Índia: Rubber Research Institute of India.

Ramos, L. M. A., Latorraca, J. V. D. F., Castor Neto, T. C., Martins, L. S., \& Severo, E. T. D. (2016). Anatomical characterization of tension wood in Hevea brasiliensis (Willd. ex A. Juss.) Mull. Arg. Revista Árvore, 40(6), 1099-1107. http://dx.doi.org/10.1590/0100-67622016000600016. 
Riyaphan, J., Phumichai, T., Neimsuwan, T., Witayakran, S., Sungsing, K., Kaveeta, R., \& Phumichai, C. (2015). Variability in chemical and mechanical properties of Pará rubber (Hevea brasiliensis) trees. ScienceAsia, 41(4), 251-258. http://dx.doi.org/10.2306/scienceasia1513-1874.2015.41.251.

SAS Institute Inc. (1999). SAS Procedures Guide. Version 8 (TSMO). Cary: SAS Institute Inc.

Santana, M. A. E., Eiras, K. M. M., \& Pastore, T. C. M. (2001). Avaliação da madeira de quatro clones de Hevea brasiliensis por meio de sua caracterização físico-mecânica. Brasil Florestal, 70, 61-68.

Santos, H. G., Jacomine, P. K. T., Anjos, L. H. C., Oliveira, V. A., Oliveira, J. B., Coelho, M. R., Lumbreras, J. F., \& Cunha, T. J. F. (Ed.), (2006). Sistema brasileiro de classificação de solos (2. ed., 306 p.). Rio de Janeiro: Embrapa Solos.

Severo, E. T. D., Oliveira Junior, E. F., Sansigolo, C. A., Rocha, C. D., \& Calonego, F. W. (2013). Properties of juvenile and mature woods of Hevea brasiliensis untapped and with tapping panels. European Journal of Wood and Wood Products, 71(6), 815-818. http://dx.doi.org/10.1007/s00107-013-0731-2.

Vidaurre, G. B., Vital, B. R., Oliveira, A. C., Oliveira, J. T. S., Moulin, J. C., Silva, J. G. M., \& Soranso, D. R. (2018). Physical and mechanical properties of juvenile. Schizolobium amazonicum Wood. Revista Árvore, 42(1), e420101. http://dx.doi.org/10.1590/1806-90882018000100001.

Viégas, I. J. M., Carmo, M., Sampaio, T., Curta, C. R. C., Alves, M. A., Pinheiro, T. E., \& Carvalho, J. G. (2015). Efeito das doses de cálcio na produção de matéria seca e na concentração de macronutrientes em plantas jovens de seringueira (Hevea spp.). Revista de Ciências Agrárias (Belém), (35), 25-40.

Zenid, G. J. (2009). Madeira: uso sustentável na construção civil. São Paulo: Instituto de Pesquisas Tecnológicas.

Contribuição dos Autores: ILL: Conceituação, Obtenção de Financiamento, Análise Formal, Escrita; RB: Curadoria de Dados; KRB: Curadoria de Dados; MLTM: Administração do Projeto; JNG: Supervisão. 